\title{
REDUNDANT VIRTUAL MACHINES MANAGEMENT IN VIRTUALIZED CLOUD PLATFORM*
}

\author{
FAN ZHANG ${ }^{\dagger}$, JUNWEI CAO JUN $^{\ddagger}, \|$, HONG CAI ${ }^{\uparrow}$, LIANCHEN LIU ${ }^{\dagger}, \S$ \\ and CHENG $\mathrm{WU}^{\dagger}, \S$ \\ ${ }^{\dagger}$ National CIMS Engineering and Research Center \\ Department of Automation, Tsinghua University \\ Beijing 100084, P. R. China \\ ${ }^{\ddagger}$ Research Institute of Information Technology \\ Tsinghua University, Beijing 100084, P. R. China \\ $\S$ Tsinghua National Laboratory for Information Science and Technology \\ Beijing 100084, P. R. China \\ IIBM China Software Development Lab \\ 1/jcao@tsinghua.edu.cn
}

Received 28 July 2010

Revised 26 September 2010

Accepted 28 January 2011

\begin{abstract}
Selecting and utilizing proper virtual machines in a virtualized cloud platform to achieve high availability, throughput, reliability, as well as low cost and makespan is very important. The importance lies in the adaptive resource provisioning to satisfy variant of workloads. An Adaptive Accessing Aware Algorithm (A5) is proposed in this paper to deal with this conflicting objective optimization problem. The main strategy of A5 is selecting adaptive upper/lower bound of service capacity to decide the time for scheduling redundant virtual machines and a Pareto-front-based multi-objective optimization method to decide the number of scheduling virtual machines. We carried out experiments in simulation, which show that A5 can achieve much higher performance improvements in four different workload testing environments, compared with other three commonly used methods.
\end{abstract}

Keywords: Pareto-front; redundancy; upper/lower bound; virtualized cloud platform; virtual machine.

\section{Introduction}

\subsection{Research background and motivation}

The advent of Cloud Computing, ${ }^{1}$ which leverages virtual clusters to extremely large scale, widely distributed, commodity-computer data centers and consistent

* Part of this work was performed when Fan Zhang was an intern at IBM China Development Lab supported by 2010-2011 IBM Ph.D. Fellowship.

"Corresponding author. 
service provisions requires reliability, availability, throughput and lower cost. However, the optimization of all these conflicting metrics is difficult. For example, improving throughput leads to scaling the cost of using more number of virtual machines.

Recently we have witnessed lots of proposals to deal with this problem. Failover clustering $^{2}$ combines one or more nodes, or servers, with two or more shared disks. If one server fails to provide service, the failover strategy migrates the running applications to other server in the disk groups. Data redundancy ${ }^{3}$ is also very popular to provide high availability solutions. Hadoop, ${ }^{4}$ an open source project and a light-weight implementation of Google File System (GFS), ${ }^{5}$ replicates each file twice to ensure availability.

The major challenges are concluded as follows.

(1) Low utilization. There is an inborn conflicting between redundancy and utilization. Redundant virtual machines (VMs) are used as replications to provide sustainable services for high availability. Low utilization problem emerges when workloads are relatively light since lots of VMs are idle. Providing a flexible strategy to effectively utilize these resources adaptively is one of the technical challenges.

(2) High cost. Replications of data require software and hardware investment and maintenance cost. Minimizing the overall cost and keeping the system availability at an acceptable level to adapt to variant system workloads is another challenge.

(3) Low reliability. The reliability of the system may not grow linearly with the redundancy of the system, which may result in extra cost. Ensuring high reliability adaptively with consideration of cost is also difficult.

An Adaptive Accessing Aware Algorithm (A5), is proposed in this work, aimed at addressing the above issues. The major contributions include:

(1) Global optimization of throughput, availability, reliability and cost awareness. Reliability and cost of different redundant VMs have different impacts on throughput and availability. A selection strategy based on achieving Pareto-front with multi-objective programming is brought in to address this global performance metrics balance problem.

(2) Adaptive accessing awareness. Arrival/departure rate of workloads influence service capacity differently. Different requests exert different pressures on system performance since the various resources they require to use and their own complexity are also different. A5 is proposed to adaptively adjust itself for this situation and automatically change its scheduling policy to optimize the objectives in (1).

(3) Adaptive upper and lower bounds of redundant VMs. When the Cloud platform is overburdened (or over idle), an adaptive threshold of choosing available redundant VMs to enrich the active redundant list (or in an opposite 
direction) is effectively calculated to take full advantage of redundant resources. We use a heuristic method to balance low service capacity with the cost of frequently schedule in redundant VMs for lower bound selection. Also, we balance high service cost with the cost of frequently scheduling out redundant VMs for upper bound selection.

\subsection{Related work}

Redundant systems ${ }^{6}$ have long been used as an effective strategy to deal with low reliability, error-prone problems not only in computational cluster systems but also in mechanical engineering, electrical system design, etc. The redundant resource allocation is an NP-hard problem, ${ }^{7-9}$ which makes it unable to be solved by polynomial algorithm. Lee et al. ${ }^{10}$ made a comprehensive comparison of two famous strategies: max-min approach and the Nakagawa and Nakashima method, to conclude the advantage and disadvantage of them. Performance of Nakagawa and Nakashima method depends strongly on initial redundant strategy, which limits its implementation in real cluster applications. Based on the max-min method, our A5 is an adaptive extension of this method, which includes objectives more than only reliability. Azaron et al. ${ }^{11}$ proposed a new methodology for the reliability optimization of a $\mathrm{k}$ dissimilar-unit (or heterogeneous) nonrepairable coldstandby redundant VMs and applied the shortest path technique in stochastic networks to evaluate the reliability. The evaluation of reliability technique is similar in A5. Different from this work, A5 tends to find a proper interval to ensure the selected active redundant system running with a highest reliability. Salazar et al. ${ }^{12}$ detailed the redundancy strategies in constrained multi-objective reliability problems and then used evolutionary algorithms (MOEA) to identify a set of optimal solutions (Pareto-front) to ensure that the selected solutions is not dominated by any other possible solutions. A5 absorbs the advantage of Pareto-front, which orders the redundant VMs based on their reliability, cost and service capacity. Most of these works are more theoretically based, and many mature methodologies can be rectified to suit for redundant cluster systems in our A5 as described above.

Other than that, research on computational resource allocation and cluster reliability awareness provides lots of solutions to deal with similar problems. Redundant strategy has been proven to be very efficient in Peer to Peer (P2P) file sharing system ${ }^{13}$ named Hyper-Chord. Different from that, our proposed A5 is not a structured architecture. Coit ${ }^{14}$ researched on many cold-standby redundancies to maximize system reliability from theory and proposed its implementation in cost-efficient engineering designs. Hong et al. ${ }^{15-17}$ also considered adaptive resource allocation problem with equal-sized task to maximize the throughput. Instead, the A5 algorithm tends to consider tasks with unequal sizes (which brings in different pressure on system performance) and uses more performance metrics to heuristically and adaptively dispatch the redundant VMs. 
Grid workflow scheduling problems for performance improvement are investigated in Refs. 18-21. Our A5 is different from these works in that it mainly deals with virtualized cloud platform, in which virtual resources are provisioned on demand. Most of the previous related works for grid or P2P resource scheduling are based on the fully utilization of all the resources. This is a very different point.

The remainder of the paper is organized as follows. Section 2 introduces the redundant virtualized cloud platform model and traditionally used max-min optimization strategy. Section 3 introduces the rationale of A5. Section 4 gives out A5 algorithm and the analysis. Abundant experiments and performance evaluation are conducted in Sec. 5 to show the efficiency of A5 over other methods. At last, some conclusions are made and future works for improvements are listed in Sec. 6.

\section{Redundant Virtualized Cloud Platform}

\subsection{Preliminaries of dynamic redundant VMs}

In Fig. 1, we depict the virtualized cloud platform built with four virtual clusters over three physical clusters. Each physical cluster consists of a number of interconnected servers, represented by the rectangular boxes with three different shadings for the three physical clusters shown. The virtual machines are implemented on the servers (physical machines). Each virtual cluster can be formed with either physical machines or VMs hosted by multiple physical clusters. The boundaries of the virtual clusters are shown with four dot/dash-line boxes. The provisioning of VMs to a virtual cluster can be dynamically done upon user demands.

Theoretical notations we use in our experiment are firstly summarized in Table 1. Figure 2 shows the workload generation scenario and sketch of our scheduling systems.

There are three lists in our experiments.

(1) Active redundant list (ActiveReduList): It holds redundant VMs that are currently in use represented by the green rectangular box in Fig. 2.

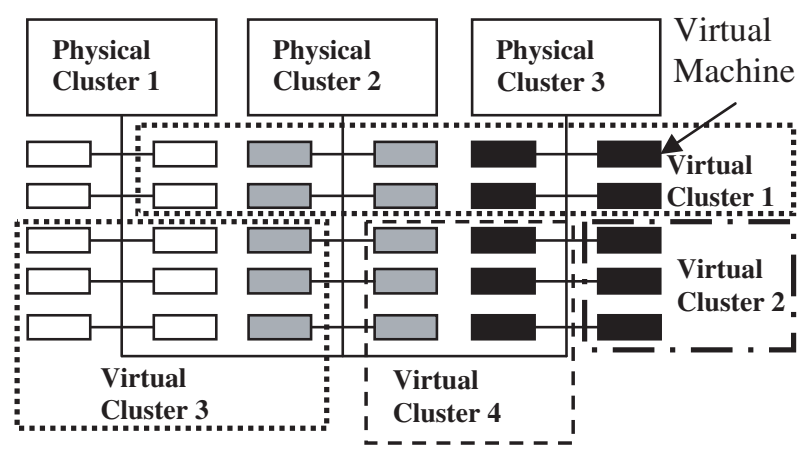

Fig. 1. A virtualized cloud platform with four virtual clusters deployed on three physical clusters. 
Table 1. Notations for use.

\begin{tabular}{ll}
\hline Notations & \multicolumn{1}{c}{ Descriptions } \\
\hline$s$ & Number of physical clusters \\
$n_{i}$ & Number of total VMs in physical cluster $i(i \in[1, s])$ \\
$m_{i}$ & Number of available VMs for physical cluster $i$ \\
$x_{i j}$ & Number of VMs in physical cluster $i$ and we choose VM $j$ to use \\
$C$ & Maximum allowable system cost \\
$c_{i j}, r_{i j}$ & Cost, weight and reliability of choosing virtual machine $j$ in \\
$R_{s}, T_{s}, C_{s}$ & physical cluster $i$ \\
$R_{i}\left(x_{i}\right), T_{i}\left(x_{i}\right) C_{i}\left(x_{i}\right)$ & Overall reliability, throughput and cost \\
\hline
\end{tabular}

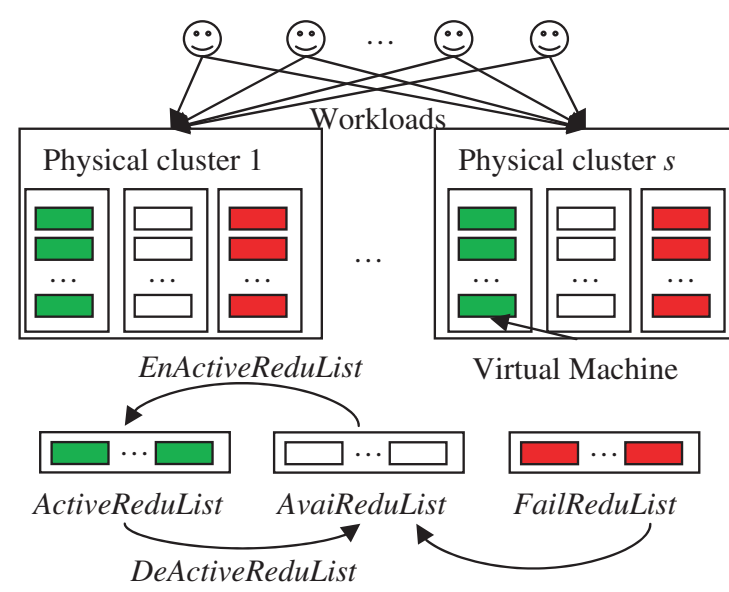

Fig. 2. Workload generation scenario and sketch of our scheduling systems.

(2) Available redundant list (AvaiReduList): It holds redundant VMs that are prepared to use represented by the white rectangular box in Fig. 2. We schedule redundant VMs into ActiveReduList from AvaiReduList when the cloud platform is overburden (EnActiveReduList) or release extra redundant VMs when it is over idle (DeActiveReduList).

(3) Failed redundant list (FailReduList): It holds VMs that are failed currently or permanently represented by the red rectangular box in Fig. 2.

Based on these analyses, we can see that the workloads generated by all terminal users feeding into physical cluster $i(i \in[1, s])$ and its redundant virtual servers $i j\left(j \in\left[1, m_{i}\right]\right)$ form a queue service model. But this queue characterizes itself in the following two aspects, which is different from the traditional queue theory.

(1) Rather than one workload have to be pended until its previous one finished its service, multi-thread processing allows all workloads arrived at one physical 
cluster could be executed concurrently, which violates the most basic premise in queue theory. ${ }^{23}$

(2) Availability of VM violates another basic premise that service provider should be always reliable. It is very common that some services are possibly to be terminated because of the potential failures of VMs.

Different from queue theory that service provider has stable service capacity $(S C)$, our system provides dynamic service capacity $S C(t)$ in that there are some reliability concerns and variable service providers' scenarios. Too high $S C$ means too many redundant VMs, which brings in cost problem. On the other hand, too low $S C$ brings in problems of low throughput.

\subsection{Max-Min approach}

The most commonly used max-min approach was first developed by RamirezMarquez et al., ${ }^{22}$ which takes into account the reliability of the whole system as the optimizing objective. It is constrained by the cost since its basic premise is that a subsystem with redundant VMs has a higher or at least an equal reliability than the original system. Based on these results, the max-min approach for redundant system can be formulated as follows (we use reliability as an example):

$$
\underset{x}{\max }\left(R_{s}\right)=\max _{x} \prod_{i}\left(R_{i}\left(x_{i}\right)\right) .
$$

Subject to

$$
\begin{gathered}
\sum_{i} \sum_{j} c_{i j} x_{i j} \leq C \\
x_{i j} \in Z^{+} .
\end{gathered}
$$

The problem of maximizing the reliability of each component subject to cost and weight constraint equals to maximizing the reliability of minimal reliable component. In this way, the objective function can be reformulated as

$$
\max _{x}\left(R_{s}\right)=\max _{x}\left\{\min _{i}\left\{1-\prod_{j}\left(1-r_{i j}\right)^{x_{i j}}\right\}\right\} .
$$

This is an integer programming problem, and there are many existing methods, such as interior point cutting plane method or branch-and-bound algorithm, to solve it. Also we can transform the constrained optimization problem into unconstrained problem by bring in a penalty function.

\section{Adaptive Accessing Aware Strategy}

\subsection{Preliminaries of $A 5$}

A5 addresses a multi-objective optimization problem, which includes four performance metrics: throughput, cost, makespan, and reliability. Detailed introduction of these metrics are given in Sec. 3.2. 


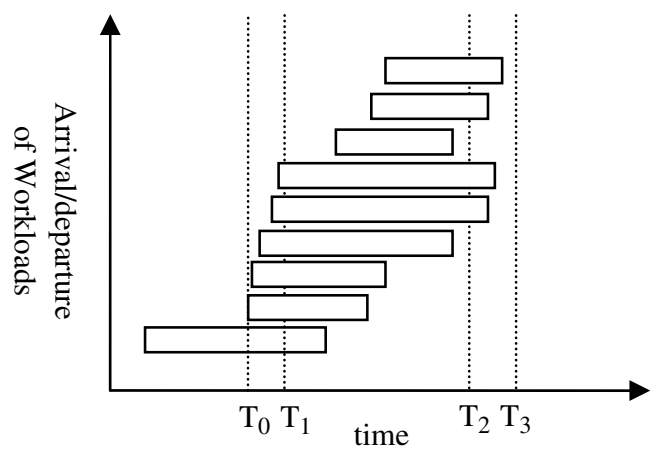

Fig. 3. Arrival and departure of workloads, the length of rectangular box represent its EET.

The main principle of A5 is demonstrated in Fig. 3. During $\left[T_{0}, T_{1}\right]$, a dense arrival rate with long Expected Execution Time (EET) requests, which leads to A5 to choose proper redundant VMs, EnActiveReduList, to distribute the workload. During $\left[T_{2}, T_{3}\right]$, a dense departure rate leads A5 to release redundant VMs, DeActiveReduList, to ensure a low cost. Deciding the time to choose appropriate VMs used is our main target. In the following sections, an upper/lower bound selection method answers the "when" problem and a Pareto-front-based multi-objective optimization scheduling answers the "how" problem.

In view of the above analysis, the following concepts and analysis are under consideration when we design redundant strategy.

(1) Real-time service capacity (SC(CurTime)). Based on Yang's work ${ }^{24}$ on service capacity in $\mathrm{P} 2 \mathrm{P}$ systems, the increase of peer nodes' join can increase system performance from $\beta$ to $\beta m$ as the replica increase $m$ times. This can be applied here straightforward since we share a similar scenario. If the current $S C$ of our platform at time $t$ is $S C(t)$, another redundant VM with service capacity $S C^{\prime}(t)$ is chosen to enlist in need, then the service capacity can be simplified as $\left(S C(t)+S C^{\prime}(t)\right)$. Similar rule can be applied in delist.

(2) Adaptive upper and lower bound of service capacity. Upper/lower bound at current time, which are denoted as UpperBound(CurTime) and LowerBound(CurTime), respectively, are used for deciding when to increase/decrease redundant VMs. Suppose the system is preferably to run at an average SC as shown in Fig. 4, if $S C$ (CurTime) > UpperBound(CurTime), it means that extra service capacity is used and we decide to do DeActiveReduList. On the other hand, if $S C$ (CurTime) < LowerBound(CurTime), more VMs are needed because of low service capacity. The major difficulty of making the decision is determining how to adaptively decide the two bounds. If UpperBound(CurTime) is too high as shown in 1, it will potentially enlist many redundant VMs until it is the time to release, which makes the system 


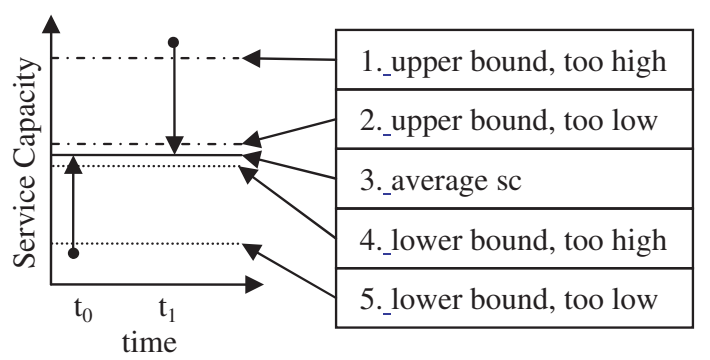

Fig. 4. Demonstration of upper/lower-bound.

runs at a very high $S C$ but leads to unnecessary running cost. On the contrary, if it is set too low as shown in 2 , the bound is easy to go over, which leads to frequently DeActiveReduList() and brings other cost overhead. Similar dilemma exists in the selection of LowerBound(CurTime). Proper and adaptive decision of the two bounds can decrease total cost and increase throughput significantly.

(3) En/De-ActiveReduList strategy. Three metrics, namely $S C$, cost, and reliability, are considered altogether. In Fig. 4, we want to choose a combination of redundant VMs (EnActiveReduList) that can lead to the $S C$ approaching a value very close to average $S C$ with low cost and high reliability. The optimization problem can be formulated like this:

$$
\left\{\min \left(\left|\operatorname{asc}-\left(S C\left(t_{0}\right)+\sum_{i=1}^{s} S C(s)\right)\right|\right), \min \left(\sum_{i=1}^{s} \operatorname{Cost}(s)\right), \max (\min (\operatorname{Re} l i(s)))\right\},
$$

where $s$ is the index of the selected redundant VMs in the available redundant list and asc denotes the average service capacity. It is a multi-objective optimization problem. DeActiveReduList can be similarly formulated as follows (suppose the current time is $t 1$ ),

$$
\left\{\min \left(\left|a s c-\left(S C\left(t_{1}\right)+\sum_{i=1}^{s} S C(s)\right)\right|\right), \min \left(\sum_{i=1}^{s} \operatorname{Cost}(s)\right), \min (\max (\operatorname{Re} l i(s)))\right\},
$$

where $s$ is the index of the selected redundant VMs in active redundant list.

(4) Pareto-front-based scheduling. Mathematically, achieving Pareto-front is defined as a multi-objective optimization problem. Supposing our bi-objective optimization problem is $\min \left(\mathbf{J}_{\mathbf{1}}(\boldsymbol{\theta}), \mathbf{J}_{\mathbf{2}}(\boldsymbol{\theta})\right)$ and we plot all $\left(\mathbf{J}_{\mathbf{1}}, \mathbf{J}_{\mathbf{2}}\right)$ pairs in Fig. 5. Intuitively we can see red dots $\left\{\ell_{1}\right\}$ are better, at least not worse, than other white dots from both $\mathbf{J}_{\mathbf{1}}$ and $\mathbf{J}_{\mathbf{2}}$. Thus they are called Pareto-front. If we remove $\left\{\ell_{1}\right\},\left\{\ell_{2}\right\}$ forms the Pareto-front of the remaining space. In this way, we 


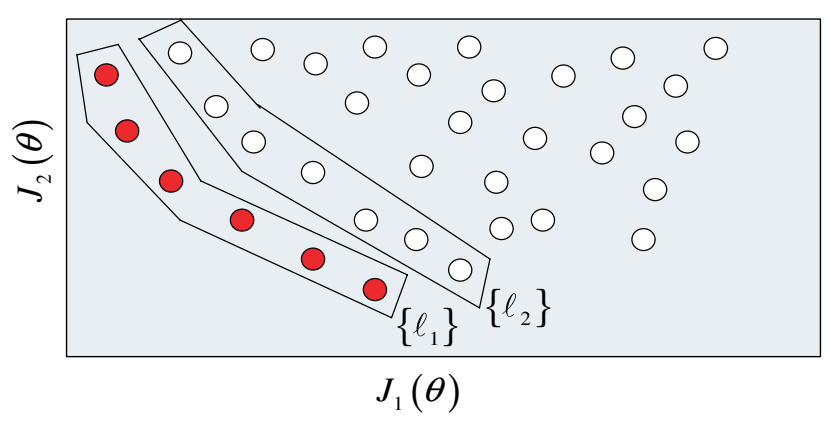

Fig. 5. Demonstration of Pareto-optimal sets.

can get a series of ordered layers $\left\{\ell_{1}\right\},\left\{\ell_{2}\right\}, \ldots$ This rule can also be similarly applied in more objectives.

\subsection{Optimization metrics analysis}

Based on the above analysis, we reformulate the optimization objectives as follows:

$$
\{\max (R(E T)), \min (M(E T)), \max (T(E T)), \min (C(E T))\},
$$

where ET, R, M,T,C are short for Experimental Time span, Reliability, Makespan, Throughput and Cost, respectively. The definition of each metric is as follows:

(a) $R(E T)$ : average $(\max (\operatorname{Reli}($ ActiveReduList $(i))$ at time $t)), i \in[1$, length (ActiveReduList) at time $t$.

(b) $M(E T)$ is defined as: $\forall t=\left[t_{0}, t_{1}\right]$, if ActiveReduList during $\mathrm{t}$ is not null, $M(T)=M(T)+t$.

(c) $T(E T)$ is defined as: Number of Workloads $/ M(T)$.

(d) $C(E T)$ is defined as: $\forall t=\left[t_{0}, t_{1}\right]$, if ActiveReduList at $t$ is not null, $\forall n$ in ActiveReduList, $C(E T)=\mathrm{C}(E T)+\operatorname{Cost}($ ActiveReduList $(n)) *\left[t_{1}-t_{0}\right] . \forall i=$ En/De-ActiveReduList()attimet', $C(E T)=C(E T)+\operatorname{SchedCost}(i)$.

\section{Explanation of the four performance metrics:}

If ActiveReduList is not empty at time $t$, the reliability of the whole system is decided by the maximum reliability of the VMs in the list. A5 tries to find an average best during the ET as shown in (a).

Makespan is defined as the aggregated timespan that at least one VM is being in use. If ActiveReduList is not empty at time $t$, then it means there are still workloads under processing. The makespan should include all this part of time as shown in (b).

Throughput is defined by the total workloads divided by the total makespan, which shows how many workloads are processed on average during the whole experimental time as shown in (c). 
In (d), we can see that the cost is composed of two parts. The first part is the service cost, which equals to how long each redundant VM $n$ is used $\left(\left[t_{1}-t_{0}\right]\right)$ and its unit cost $(\operatorname{Cost}($ ActiveReduList $(n)))$. The second part is the scheduling cost, which includes scheduling in/out cost of redundant system $i$ to/from ActiveReduList $(\operatorname{SchedCost}(i))$.

\section{Adaptive Accessing Aware Algorithm}

Algorithm 1 below is the main body of A5. It uses reliability, cost, throughput, and makespan based on definition in Sec. 3. At the beginning of the experiment, the workload increases with large bulks of requests, which decrease service capacity under the LowerBound(CurTime), EnActiveReduList() is invoked to increase service capacity. As the experiment goes on, more requests are finished. This alleviate service capacity above UpperBound(CurTime), A5 invokes DeActiveReduList() to avoid too much cost problem. How to find a proper pair of UpperBound/LowerBound dynamically is demonstrated in Algorithm 2, then the strategies of enriching/releasing redundant VMs are shown in Algorithm 3.

\subsection{A5 algorithm}

\begin{tabular}{|c|c|c|}
\hline & & Algorithm 1. A5 Algorithm \\
\hline & $\begin{array}{c}1 . \\
2 . \\
\\
3 . \\
4 . \\
5 . \\
6 . \\
7 . \\
8 . \\
9 . \\
10 . \\
11 . \\
12 . \\
13 . \\
14 . \\
15 . \\
16 .\end{array}$ & 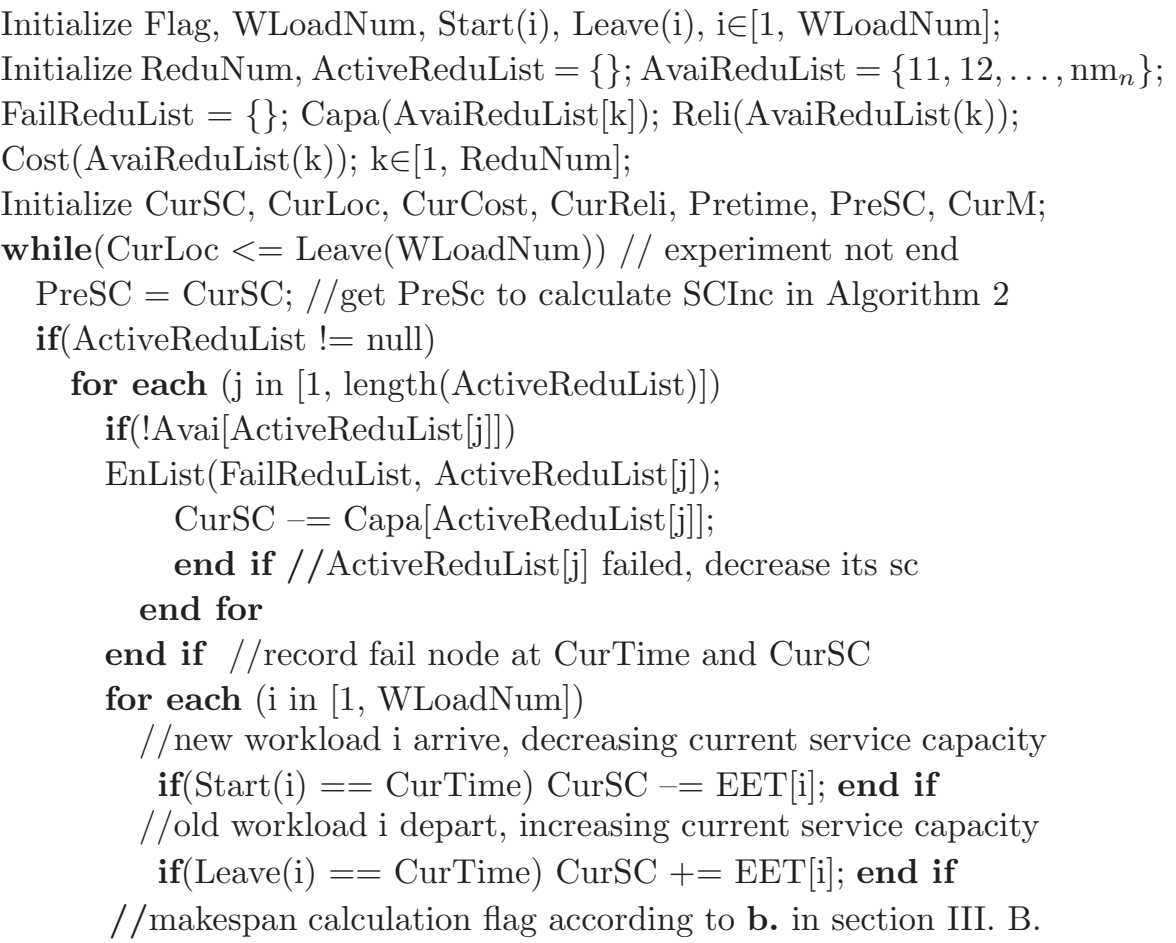 \\
\hline
\end{tabular}




\begin{tabular}{|c|c|}
\hline & Algorithm 1. A5 Algorithm (Continued) \\
\hline 17. & if $($ CurTime $>$ Start(i) \& \& CurTime $<$ Leave(i)) Flag $=1$; end if \\
\hline 18. & end for //find next start or leave request and their influence on CurSC \\
\hline 19. & while(CurSC < LowerBound(CurTime)) // invoke algorithm 2 \\
\hline 20. & EnActiveReduList(); //Overload, invoke algorithm 3 \\
\hline 21. & end while \\
\hline 22. & while(CurSC > UpperBound(CurTime)) // invoke algorithm 2 \\
\hline 23. & DeActiveReduList(); //over idle, invoke algorithm 3 \\
\hline 24. & end while \\
\hline 25. & $\begin{array}{l}\text { if }(\text { Flag }==1) \text { CurM }+=1 / \text { CurSC; end if //makespan calculation } \\
\text { //CurLoc goes for one step unit and calculate the time consuming }\end{array}$ \\
\hline 26. & CurLoc ++ ; PreTime $=$ CurTime; CurTime $+=1 /$ CurSC; \\
\hline 27. & for each ( $\mathrm{j}$ in $[1$, length(ActiveReduList $)])$ \\
\hline 28. & TotalCost $+=$ Cost $($ ActiveReduList $[\mathrm{j}]) /$ CurSC \\
\hline 29. & end for //Find TotalCost and Total Reliability currently \\
\hline 30. & TotalReli $+=\max ($ Reli $($ ActiveReduList $))$ \\
\hline & //according to a. in section III.B. \\
\hline 31. & end while \\
\hline 32. & MakeSpan = CurM; //summarize to get makespan and throughput \\
\hline 33. & Throughput=WLoadNum/MakeSpan;//according to $\mathbf{c}$. in section III.B \\
\hline
\end{tabular}

Algorithm 2. Upper/LowerBound(CurTime)

34. TimeInc $=$ CurTime - Pretime;

35. $\mathrm{SCInc}=\mathrm{CurSC}-\mathrm{PreSC}$;

//If SCInc $<0$, SC decreased, Upper/Lower bound decrease accordingly, else Upper/Lower bound increase accordingly

36. UpperBound $=$ UpperBound + SCInc/ $($ TimeInc $*$ f $)$;

37. $\quad$ LowerBound $=$ LowerBound + SCInc/ (TimeInc*f);

38. $\quad$ Asc $=($ UpperBound + LowerBound $) / 2$;

39. end

Algorithm 3. En/De-ActiveReduList()

40. $\{\{1\},\{2\}, \ldots,\{\mathrm{j}\} /\{\mathrm{k}\}\}=$ ParetoFront(Avai/ActiveReduList)

41. for each $(\{n\}$ in $\{\{1\}, \ldots,\{j\} /\{k\}\})$

42. if $(\{n\} !=$ null $) / /\{n\}$ contains the nodes in $n^{\text {th }}$ layer of pareto front 


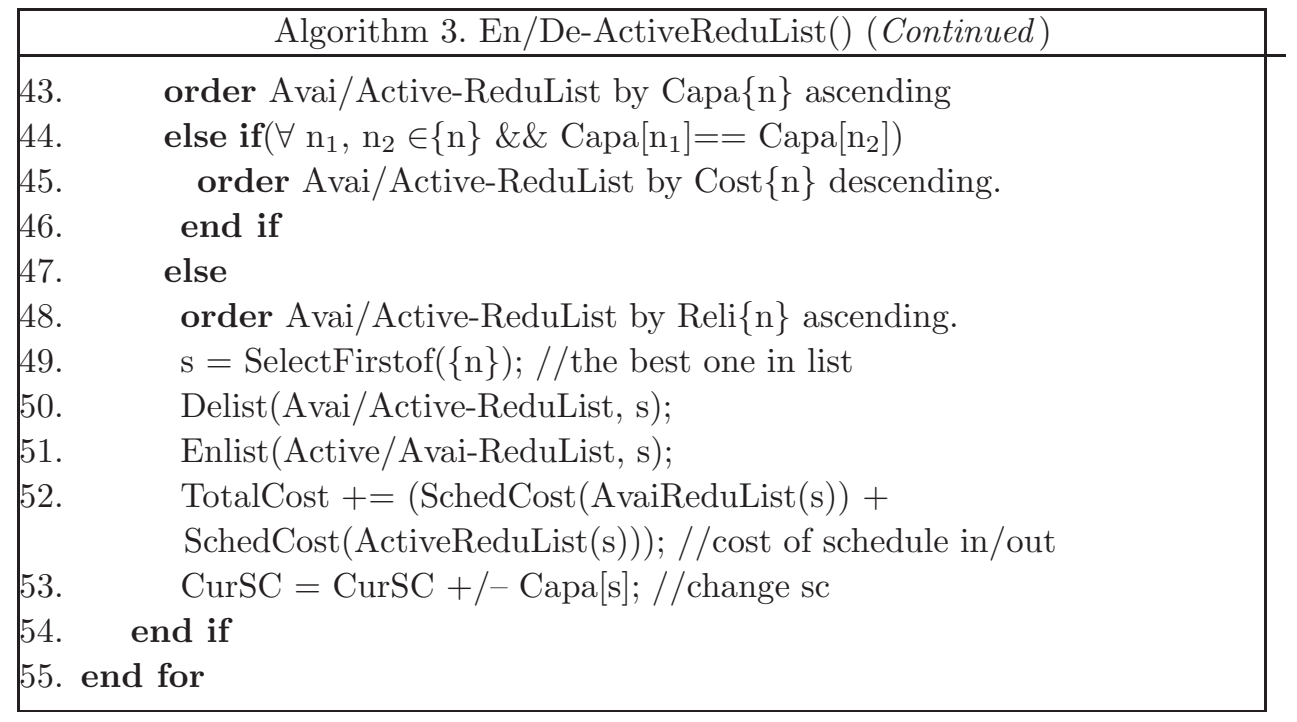

$\{1\},\{2\}, \ldots,\{j\} /\{k\}$ are ordered layers of Avai/Active-ReduList respectively based on (5) in Sec. 3.1. For the redundant VMs of one Pareto-front layer, the selection are ordered by service capacity, cost and reliability. In this way, we can guarantee an optimum selection of preferable suitable VMs.

A flowchart in Fig. 6 is used to illustrate the application procedure of our A5 algorithm.

\subsection{Algorithm analysis}

In our algorithm, the current time is CurTime and the service capacity is CurSC. Each CurLoc goes one step forward and the time it takes is different because of different CurSC. There are three points that affect CurSC.

(1) Fail of running VM $\boldsymbol{i}$. If VM $i$ fails to provide service, the system would decrease service capacity by Capa $[i]$, which is shown in line 6-13. To make things simpler, we unify the unit of $C a p a[i]$ into $C u r S C$ to make them directly

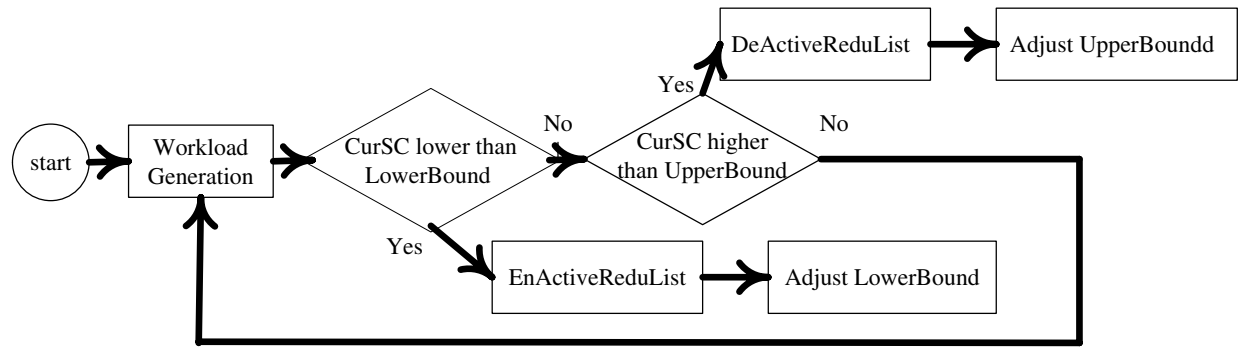

Fig. 6. A flowchart to illustrate the algorithm. 
addable/subtractable as shown in line 10 of Algorithm 1 and line 53 of Algorithm 3.

(2) Arrive/Leave of requests. Newly arrived requests decrease service capacity depending on their EET. The algorithm simulates this scenario by automatically generating workloads with different EET to show their different impacts on service capacity. Similar with (1), we also unify the unit of requests' impact EET into service capacity to make then addable/subtractable as shown in line 15, 16 of Algorithm 1.

(3) The increase/decrease of redundant VM list. As shown in algorithm 3, if we schedule redundant VMs into ActiveReduList, the CurSC would increase by Capa $[s]$ and vice versa. We bring in the concept of Pareto-front to order the performance metrics, namely sc, cost and reliability as shown in Algorithm 3, into different layers (line 40, Algorithm 3). We then select from the first layer on, which contains VMs with better sc (line 43), cost (line 45) or reliability (line 48). This is in the sense of pareto optimal as the red dots compared with the white dots in Fig. 5 .

The decision of when to increase/decrease redundant VMs depends on LowerBound/UpperBound. The two bounds are decided by the rate of fluctuation of service capacity. As shown in Algorithm 2. Here we only introduce the situation that should increase redundant VMs. Intuitively, LowerBound should decrease, at least not increase, as $C u r S C$ decreased, but it should not be faster than, or even equal to the rate of CurSC since if so, redundant VMs would never be used. We heuristically divide the rate by $\mathrm{f}(\mathrm{f}>1)$ in line 36 and 37 , to deal with this problem. After the setting of LowerBound, UpperBound changes with the same ratio. Abundant experiments show that the selection of $\mathrm{f}$ differs with the four experimental environments.

\section{Performance Evaluation}

Simulation test bed is set with 100 redundant VMs that can provide service simultaneously. The service capacity is strongly dependent on CPU rate and network condition which is similar in work. ${ }^{25}$ We then generated cost and reliability of each redundant system that is proportional to its service capacity, which is reasonable in real scenarios. To make this simulation more practical, the arrival frequency of each workload is a long range that shows their different impacts on the performance metrics. Besides that, EET is properly randomly generated based on the service capacity and reliability of each redundant VM as well as their arrival frequencies.

The workloads can be categorized into four groups. In the first group, the workload has low frequency and low EET (LFLE), which means each request comes very occasionally and each one consumes a minor fraction of resource. The second group has workload with high frequency and low EET (HFLE). The third group has workload with low frequency and high EET (LFHE), which means the interval arrival time of two workloads are long but each request is resource consuming. The 
last group has workload with high frequency and high EET (HFHE), which give the server most pressure.

The experiments are carried out using two computers separately. One is Windows XP with Intel Core 2 Duo T5870, 2G RAM and the other is Windows XP with Intel Pentium D $2.8 \mathrm{GHz}, 2 \mathrm{G}$ RAM. The final results are averaged by 10 experimental results.

\subsection{Performance of adaptive upper/lower bound}

Adaptive upper/lower bound selection is an effective way to automatically adjust redundant strategy to deal with the frequently changing workloads. The workloads are generated in uniform distribution in our experiment and are set in Table 2.

Here we discuss a scenario that cost and reliability are proportionally correlated with service capacity. Service capacities of all redundant VMs are normalized to be at a mean value of 100 with standard deviation of 20 , which is concluded by our experiments with testing a same application on different servers. The cost is normalized with the same mean of service capacity, but with standard deviation of 50 . The reliabilities of them are set with a mean of $90 \%$ and a standard deviation of $10 \%$. We performed many fixed upper/lower bound (generally upper bound is set with a mean of 1.8, lower bound is set with a mean of 1.6 , and average $S C$ is set with a mean of 1.7) experiments and averaged the results. The initial setting of the adaptive strategy is the same with the mean of the fixed setting. The results are shown in Fig. 7.

We can see from the Fig. 7 that with a fixed (non-adaptive) upper/lower bound choice, the performance in all four experiments are worse than adaptive selection. The improvements are even noticeable in cost because of line 45 in Algorithm 3 since cost is the second most important criterion for redundant strategy. The most important one is the deviation from the average $S C$ (the first item of (3) and (4)), which is decisive and a guarantee that A5 can make sure the average $S C$ not too high or too low, and it leads to better performance in the four metrics under the four experimental environments.

\subsection{Comparison with other methods}

We first compare the A5 algorithm with other methods on the four performance metrics. These methods include traditional Max-Min algorithm (MM) with fixed

Table 2. Workload settings.

\begin{tabular}{lcl}
\hline & Inter-arrival time & \multicolumn{1}{c}{ EET } \\
\hline LFLE & $\mathrm{U}(25,30)$ & $\mathrm{U}(3,5)$ \\
LFHE & $\mathrm{U}(25,30)$ & $\mathrm{U}(35,50)$ \\
HFLE & $\mathrm{U}(3,5)$ & $\mathrm{U}(3,5)$ \\
HFHE & $\mathrm{U}(3,5)$ & $\mathrm{U}(35,50)$ \\
\hline
\end{tabular}



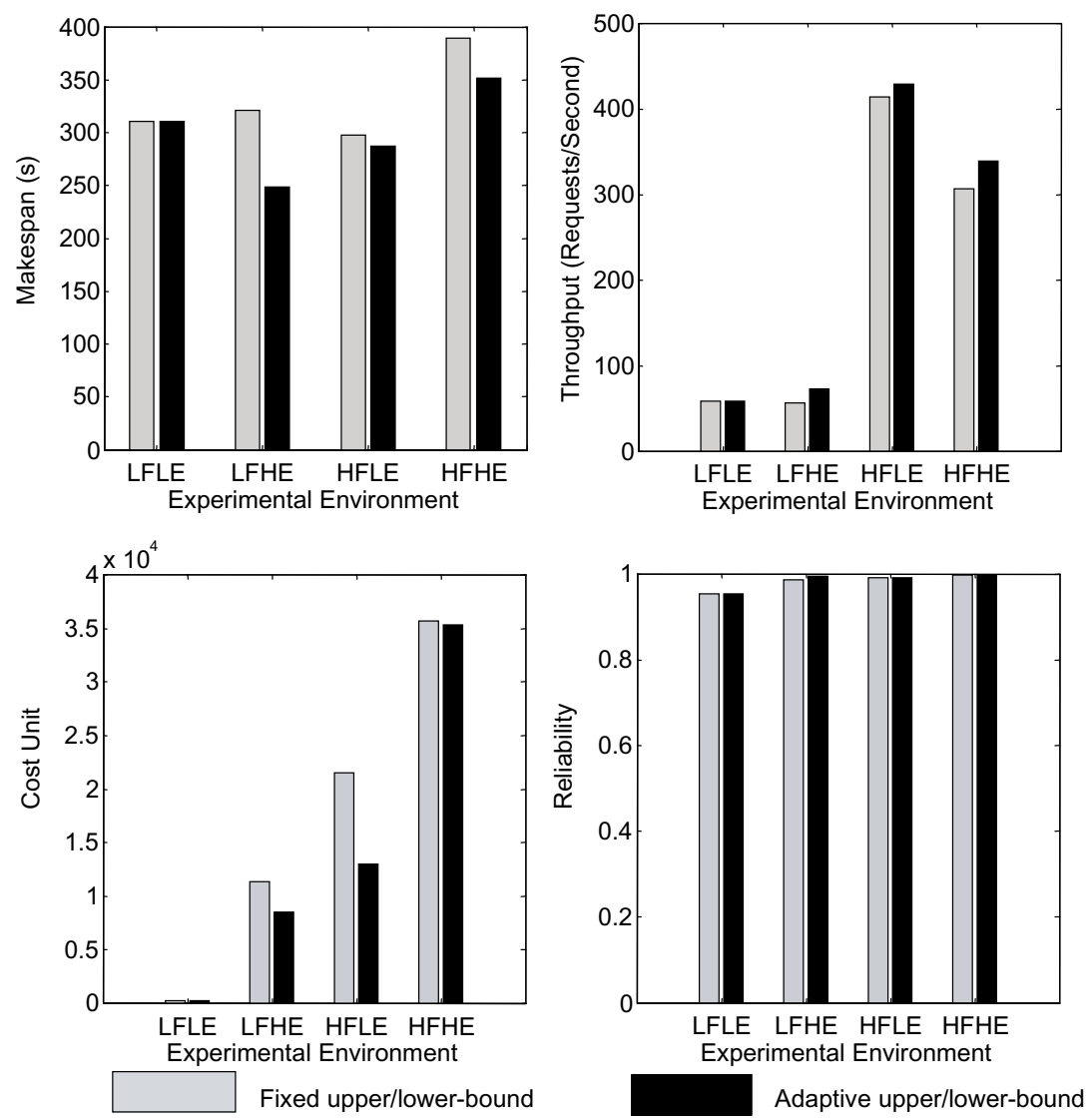

Fig. 7. Fixed (non-adaptive) versus adaptive upper/lower bound.

upper/lower-bound, Random Selection of redundant VMs with Fixed upper/lowerBound (RSFB), Random Selection of redundant VMs with Adaptive upper/lowerBound (RSAB). The workload is amplified largely in LFLE scenario in order to make the experiments identifiable and trackable. This leads to the makespan possibly larger than that in other experimental environments. We consider the priority of performance metrics in an order of throughput, cost and reliability. This leads to a possible lower reliability of A5 than other methods a little bit, which is ignorable. The experimental results are shown in Fig. 8.

We can conclude the experiments as follows.

(1) In LFLE, workloads exert no pressure on the system, thus the performance of each method is similar. Optimization strategy has no noticeable effect when the service capacity is much larger than the impact of the workloads. This advantage of A5 becomes more obvious as the system works in a much higher workload scenario. 


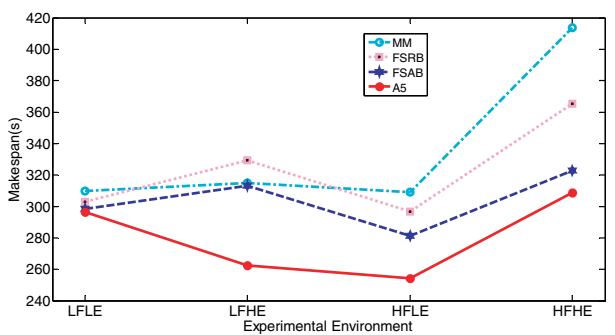

(a) Comparison over makespan

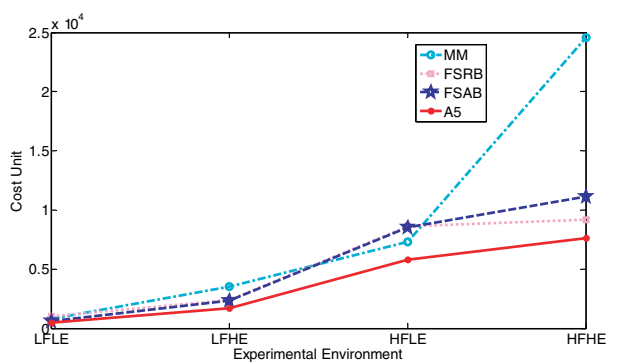

(c) Comparison over cost unit

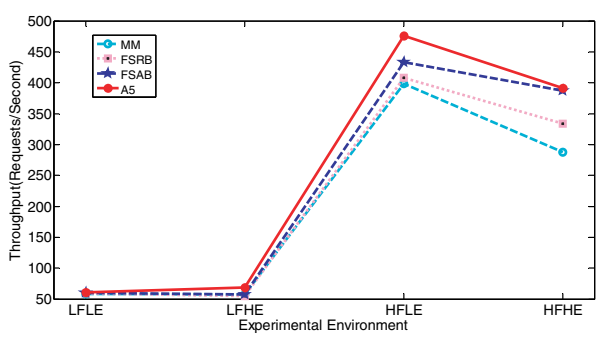

(b) Comparison over throughput

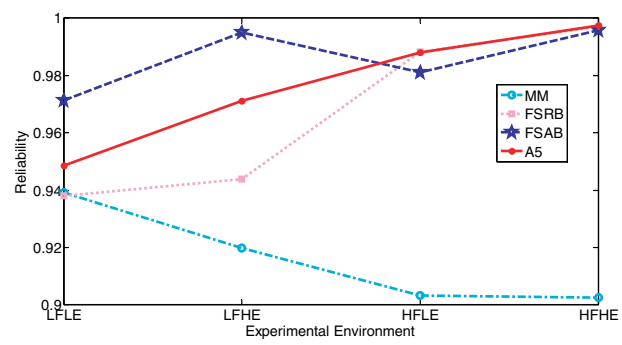

(d) Comparison over reliability

Fig. 8. Comparison of different methods.

(2) Random selection strategy of redundant VMs, which simplify the process, is at the cost of larger makespan, higher cost to schedule the improper redundant VMs and lower throughput. This problem becomes even worse as the workload becomes heavier (with higher arrival rate and longer EET). A5 save more than $70 \%$ cost and nearly $36.8 \%$ of makespan than that of MM. Compared with other methods, the number is not so much, but the improvement is very clear in all the figures.

(3) Adaptive upper/lower-bound selection is better than fixed choice. We can conclude that from Figs. 7 and 8. When this adaptive bound is combined with a proper selection of the redundant resources (A5), the effects are even better.

\section{Conclusion and Future Work}

In this paper, we proposed A5, An Adaptive Accessing Aware Algorithm, to improve throughput and reliability as well as reduce makespan and scheduling cost of a Virtualized Cloud platform consists of many virtual machine resources. The adaptivity is shown not only in upper/lower-bound selection adaptively to decide when to schedule in/out redundant VMs list, but also in Pareto-front-based redundant VMs selection adaptively to decide how to execute that schedule. Abundant 
experiments show that this method outperform other strategies in the above performance metrics.

In future research, we will consider investigating different patterns of arrival rate of workloads with uniform, exponential and normal distributions. Also we will consider the scenario of using predictive methods to ensure even better performance. Last but not the least, we intend to implement this algorithm into the real data centers in Tsinghua University with 128 nodes, which will be able to provide sufficient service capacity to validate the effectiveness of this algorithm.

\section{Acknowledgments}

This work was supported by National Science Foundation of China (grant No. 60803017) and Ministry of Science and Technology of China under National 973 Basic Research Program (grants No. 2011CB302505 and No. 2011CB302805) and National 863 High-tech R\&D Program (grants No. 2008AA01Z118 and No. 2008BAH32B03). We thank IBM for 2010-2011 IBM Ph.D. Fellowship Award.

\section{References}

1. Armbrust M., Fox A., Griffith R., Joseph A. D. et al., Above the Clouds: A Berkeley View of Cloud Computing, Univ. of California, Berkerley, Berkerley, CA, Technical Report No. UCB/EECS-2009-28, 2009.

2. Zhou Y., Chen P. M., Li K., Fast cluster failover using virtual memory-mapped communication, in Proc. SCXY'99, Rhodes, Greece, pp. 373-382, 1999.

3. Levitin G., Lisnianski A., Ben-Haim H., Elmakis D., Redundancy optimization for series-parallel multi-state systems, IEEE Transactions on Reliability 47:165-172, 1998.

4. The Hadoop project website. [Online]. Available at: http://hadoop.apache.org/core/.

5. Sanjay G., Howard G., Shun-Tak L., The Google file system, ACM SIGOPS Operating Systems Review 37:29-43, 2003.

6. Ebeling C., An Introduction to Reliability and Maintainability Engineering, McGrawHill Science/Engineering/Math, New York, 1996.

7. Kuo W., Hwang C. L., Tillman F. A., A note on heuristic method for in optimal system reliability, IEEE Transactions on Reliability 27:320-324, 1978.

8. Kuo W., Prasad V. R., An annotated overview of system-reliability optimization, IEEE Transactions on Reliability 49:176-191, 2000.

9. Kuo W., Prasad V. R., Tillman F. A., Hwang C. L., Optimal Reliability Design: Fundamentals and Application, Cambridge University Press, Cambridge, 2001.

10. Lee H., Kuon W., Ha C., Comparison of max-min approach and NN method for reliability optimization of series-parellel system, J. Systems Science and Systems Engineering 12:39-48, 2003.

11. Azaron A., Katagiri H., Kato K., Sakawa M., A multi-objective discrete reliability optimization problem for dissimilar-unit standby systems, OR Spectrum 29:235-257, 2007.

12. Salazar D., Roccob C. M., Galvan B. J., Optimization of constrained multipleobjective reliability problems using evolutionary algorithms, Reliability Engineering and System Safety 91:1057-1070, 2006.

13. Flocchini P., Nayak A., Xie M., Enhancing Peer-to-Peer systems through redundancy, IEEE Journal on Selected Areas in Communications 25, 2007. 
14. Coit D. W., Maxmization of system reliability with a choice of redundancy strategies, IIE Transactions, 35:535-543, 2003.

15. Hong B., Prasanna V. K., Bandwidth-Aware resource allocation for computing independent tasks in heterogeneous computing systems, in Proc. PODC'03, pp. 539-546, 2003.

16. Hong B., Prasanna V. K., Distributed adaptive task allocation in heterogeneous computing environments to maximize throughput, in Proc. IPDPS'04, pp. 539-546, pp. 52-60, 2004.

17. Hong B., Prasanna V. K., Adaptive allocation of independent tasks to maximize throughput, IEEE Transactions on Parallel and Distributed Systems 18:1420-1435, 2007.

18. Zhao C., Cao J., Wu H., Zhang F., Cost estimation of advance reservations over queued jobs: a quantitative study, Int. J. Mod. Simul. Scientific Computing 1(3):317$332,2010$.

19. Zhang F., Cao J., Liu L., Wu C., Fast autotuning configurations of parameters in distributed computing systems using ordinal optimization, Proc. 38th International Conference on Parallel Processing Workshops, Vienna, Austria, pp. 190-197, 2009.

20. Zhang F., Cao J., Liu L., Wu C., Qualification evaluation in virtual organizations based on fuzzy analytic hierarchy process, Proc. 7th Int. Conf. on Grid and Cooperative Computing, Shenzhen, China, pp. 539-547, 2008.

21. Cao J., Jarvis S. A., Saini S., Nudd G. R., GridFlow: workflow management for grid computing, Proc. 3rd IEEE/ACM Int. Symp. on Cluster Computing and the Grid, Tokyo, Japan, pp. 198-205, 2003.

22. Rramirez-Marquez J. E., Coit D. W., Konak A., Redundancy allocation for seriesparallel systems using a max-min Approach, IIE Transactions 36:891-898, 2004.

23. Kleinrock L., Queueing Systems. Volume 1: Theory, John Wiley and Sons, 1975.

24. Yang X., Veciana G. D., Service capacity of peer to peer networks, in Proc. INFOCOM'04, pp. 2242-2252, 2004.

25. Zhang W., Cao J., Zhong Y., Liu L., Wu C., An integrated resource management and scheduling system for grid data streaming applications, in Proc. GRID'08, pp. 258$265,2008$. 